\title{
Protagonistes de l’immunité innée dans les infections à salmonelles
} diverses maladies allant de la simple gastroentérite aux formes plus graves telles que la fièvre typhoïde, sont responsables aujourd'hui Laurent Salez, Danielle Malo encore de 600000 morts par an à travers le monde. L'élevage intensif d'animaux parfois porteurs de souches microbiennes infectieuses, ainsi que l'utilisation systématique et démesurée des antibiotiques ont permis à Salmonella et, bien sûr, à d'autres micro-organismes pathogènes, de développer des multirésistances et de poser à nouveau un réel problème de santé publique. Seize millions de personnes à travers le monde sont porteuses de diverses formes de Salmonella ; cependant, il est maintenant prouvé que les principaux sérotypes à l'origine des épidémies apparues dans les années 1980 et 1990 sont les formes les moins mortelles chez l'homme. Les recherches des 50 dernières années ont permis de mieux comprendre la physiopathologie des infections à salmonelles, notamment grâce à l'utilisation du modèle murin par approche génétique. Cet article se propose d'analyser les gènes exprimés parl'hôte, qu'ils soient humains ou murins, lors des premiers moments de l'infection. <

Salmonella, quatrième micro-organisme pathogène en importance après Yersinia, Escherichia et Shigella dans le genre entérobactéries, est un agent infectieux intracellulaire facultatif ; il a été isolé pour la première fois en 1885 par Daniel Salmon, vétérinaire américain. Depuis lors, plus de 2463 sérotypes différents ont été décrits, chacun d'entre eux étant caractérisé par une formule antigénique propre [1]. Salmonella typhi et Salmonella paratyphi sont des pathogènes humains à l'origine de fièvres typhoïdes, infections qui posent un problème de santé publique majeur dans des régions du monde où il y a peu, voire aucun, accès à l'eau potable

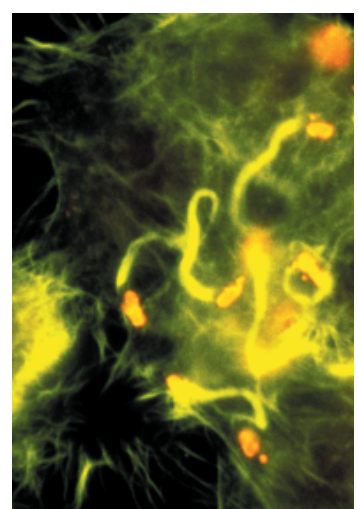

et où le traitement des eaux usées demeure insatisfaisant. La forte morbidité de ces infections et l'apparition de résistances importantes a rendu difficiles les traitements par antibiotiques classiques. Salmonella enteritidis et

Salmonella typhimurium, autres sérotypes de l'espèce Salmonella enterica, peuvent infecter des hôtes très variés (volaille, homme, souris, reptiles, amphibiens); ils causent une gastro-entérite qui, dans la plupart des cas, se résorbe sans médication antibiotique.

Les infections à Salmonella empruntent généralement la voie digestive. Chez la souris, les salmonelles provoquent une maladie systémique accompagnée de symptômes semblables à ceux de la fièvre typhoïde chez l'homme, et ce indépendamment de la voie d'infection. Classiquement, la cinétique d'infection chez la souris se caractérise par quatre phases. La première se traduit par l'élimination rapide des bactéries sériques. Durant la semaine suivant l'infection, Salmonella se réplique activement au sein des cellules phagocytaires. Cette phase précède une phase en plateau caractérisée par la 
mise en place de la reconnaissance de PAMP (pathogen-associated molecular pattern, des motifs moléculaires spécifiques de certains pathogènes) par les cellules de la lignée phagocytaire mononucléée. II en résulte la production de nombreuses cytokines (tumor necrosis factor $\alpha$, interleukines 1,6 et 12 , interféron $\gamma$ ) et une infiltration massive de monocytes et de polynucléaires neutrophiles dans les sites inflammatoires. À la quatrième phase de l'infection s'installe la défense inflammatoire dite acquise, c'est-à-dire faisant intervenir les cellules $T$ et $B$, ainsi que les facteurs humoraux qui en découlent.

Les gènes de l'hôte présentés ici, pour certains déjà connus pour d'autres fonctions, pour d'autres nouvellement découverts, suscitent un intérêt particulier si l'on veut comprendre les relations hôte-pathogène établies pendant les premières phases de l'infection (c'est-à-dire au cours de la défense innée). En outre, d'excellentes analyses synoptiques offrent un panorama complet des gènes de virulence de Salmonella, lesquels, exprimés par les pathogènes invasifs, leur confèrent un mode de résistance spécifique contre les mécanismes de défense innée [2].

\section{Reconnaissance du pathogène}

\section{CD14 et LBP}

Rappelons que Salmonella est une bactérie à Gram négatif, c'est-à-dire que sa paroi externe exprime le LPS (lipopolysaccharide), molécule fortement immunogène. La LBP (LPS-binding protein) est une protéine sérique dont la principale caractéristique est de présenter le LPS sous la forme monomérique au récepteur CD14, sa forme libre en solution étant micellaire. Les premiers travaux ayant mis en évidence le rôle de la LBP lors d'infections à Salmonella typhimurium le décrivent comme nécessaire pour qu'une réaction inflammatoire efficace se produise et contribue à éliminer le pathogène invasif [3]. Le récepteur $C D 14$, réparti majoritairement sur les monocytes et les macrophages, est le récepteur du LPS par excellence. Présent dans l'organisme sous forme membranaire (mCD14) et soluble ( $s C D 14)$, il possède en son domaine carboxyterminal un site de fixation à un groupement GPI (phosphosylphosphatidylinositol) favorisant son ancrage à la membrane cellulaire, mais sans la traverser, interdisant tout contact avec le milieu cytoplasmique et donc toute interaction moléculaire permettant la transduction du signal en aval.

La fixation du LPS à la cellule ne provoque pas d'activation cellulaire immédiate. Le laps de temps nécessaire à l'activation (15 à 30 minutes) s'explique par la nécessité d'internaliser le complexe LPS/CD14. Certaines études montrent que le blocage de la fusion endosomique ou de l'internalisation provoque une rupture dans la signalisation induite par le LPS [4]. Bien que ce mécanisme soit encore mal décrit, il a été montré que le LPS monomérique est transporté dans la cellule vers l'appareil de Golgi, puis qu'il active la cellule. II semble que le LPS particulaire (c'est-à-dire celui véhiculé par un pathogène) et le LPS micellaire (agrégé) soient, quant à eux, transportés vers le lysosome sans activer la cellule, bien que la voie empruntée demeure celle du CD14 [4].

\section{TLR4}

Jusqu'à la fin des années 1990, on ne pouvait expliquer les raisons pour lesquelles certaines lignées de souris telles que les $\mathrm{C} 3 \mathrm{H} / \mathrm{HeJ}$ et les $\mathrm{C} 57 \mathrm{BL} / 10 \mathrm{ScC}$ r possèdent une protection naturelle en cas d'injection massive de LPS [5]. A contrario, on ne comprenait pas mieux pourquoi les souris $\mathrm{C} 3 \mathrm{H} / \mathrm{HeJ}$ apparaissaient plus vulnérables que les souris $\mathrm{C} 3 \mathrm{H} / \mathrm{HeN}, \mathrm{C} 3 \mathrm{H} /$ Stet ou $\mathrm{C} 3 \mathrm{H} / \mathrm{Bi}$ à une infection à Salmonella [6].

La réponse fut apportée par des expériences de clonage positionnel qui mirent en évidence la présence d'une protéine transmembranaire orthologue de la protéine Toll, décrite initialement dans le développement embryonnaire de la drosophile [7]. La description d'une nouvelle protéine, TLR4 (Toll-like receptor 4 ), permit de comprendre comment le complexe LPS/LBP peut engendrer un message intracellulaire via le récepteur CD14. Ainsi, on sait désormais pourquoi certaines lignées murines sont naturellement résistantes au LPS ou sensibles aux infections par les bactéries à Gram négatif $[8,9]$.

TLR4 est une protéine dotée d'un domaine transmembranaire, d'un domaine extracellulaire riche en leucine et d'un domaine intracellulaire homologue au domaine intracellulaire du récepteur de I'ILl (domaine TIR). Le gène TLR4 est un représentant d'une famille de 12 gènes chez la souris et de 13 chez l'homme. Chacun d'entre eux possèdent un panel de ligands propres et, pour certains, la dimérisation - homologue ou hétérologue est un prérequis pour induire un message intracellulaire caractéristique [10]. La fixation du complexe LPS/LBP au complexe CD14/TLR4 (en coopération avec MD2, une protéine périmembranaire) produit l'activation de deux voies de signalisation distinctes et partiellement redondantes (Figure 1). Le TLR4 n'exerce pas seulement un rôle de reconnaissance du pathogène, il intervient également dans la phagocytose de bactéries à Gram négatif et positif. Cet effet est réglé par la cascade de signalisation MyD88/IRAK4/p38 et est associé à une induction du scavenger receptor [14].

Chez l'homme, plusieurs défauts génétiques de l'activation de NFKB ont été impliqués dans la survenue d'infections bactériennes récurrentes et concernent des gènes tels que IRAK4 [15], NEMO, à l'origine de la sous-unité régulatrice du complexe IKK [16], et $/ \kappa B \alpha$ [17]. Les gènes $N E M O$ et $/ \kappa B \alpha$ sont mutés chez les patients atteints de dysplasie ectodermique anhidrotique avec immunodéficience. Ces patients présentent un spectre d'infections étendu, reflétant le rôle central de NFKB dans l'immunité innée.

\section{TLR5}

Contrairement à TLR4 qui possède plusieurs ligands différents, TLR5 présente une affinité exclusive pour la flagelline 
(Figure 1). Cette protéine, exprimée dans les flagelles bactériens, présente un pouvoir très immunogène, comme en témoignent ses capacités d'activation du système phagocytaire mononucléé [18] et des cellules épithéliales de la muqueuse intestinale [19]. TLR5 induit un message transducteur similaire à TLR4, puisque la voie empruntée mobilise NFKB et induit la production de cytokines pro-inflammatoires telles que le TNF $\alpha$ et I'IL6 [18]. In vivo, TIr5 pourrait aussi jouer un rôle important dans la résistance à l'infection à Salmonella typhimurium, comme le démontrent des études de liaisons génétiques chez la souris [20].

\section{Destruction du pathogène}

\section{NRAMP 1}

La fin des années 1970 a été marquée par la localisation de trois locus de résistance à trois pathogènes différents

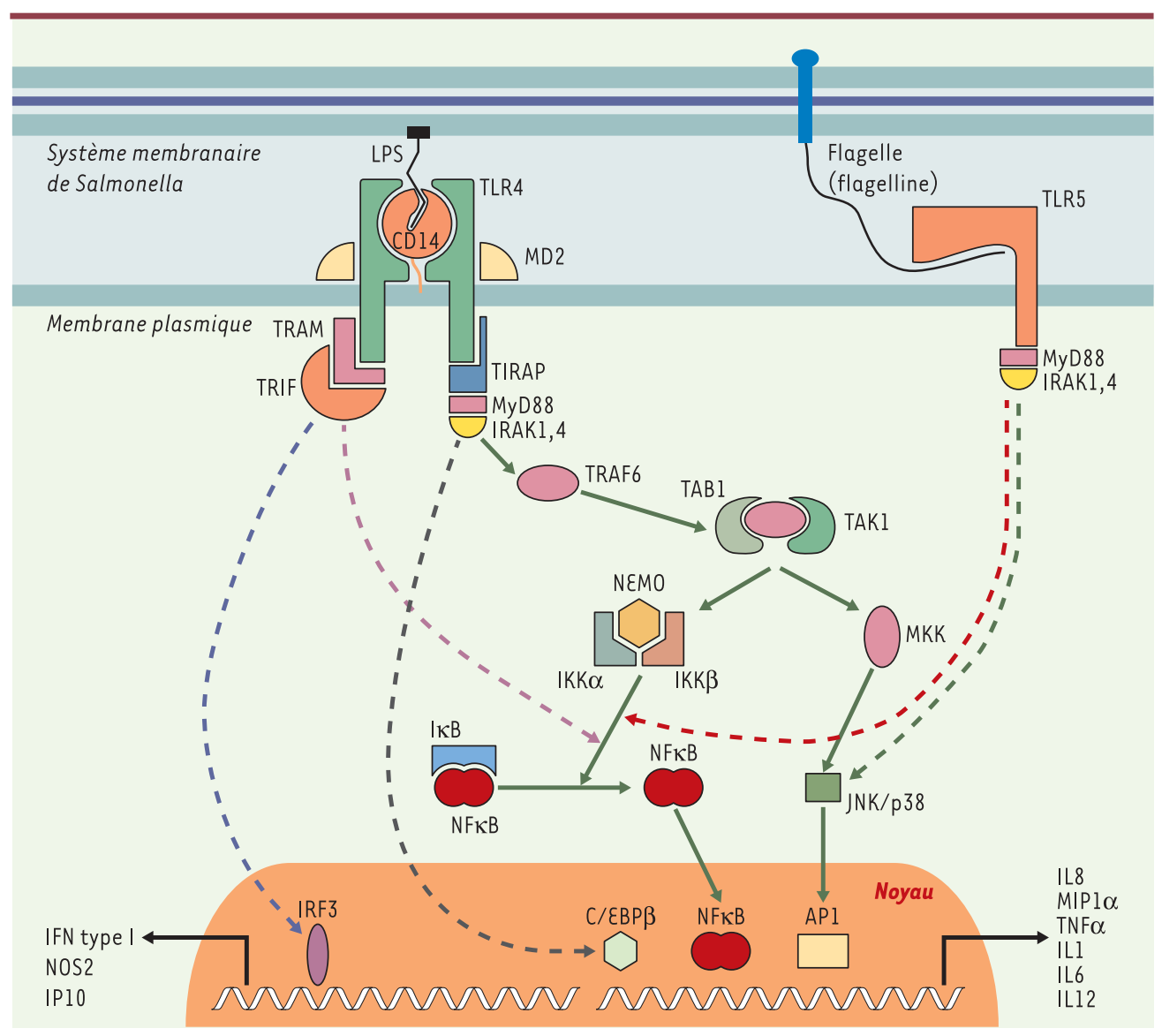

Figure 1. Interactions hôtepathogène lors de la reconnaissance de Salmonella par le système phagocytaire. L'activation non spécifique par Salmonella est déclenchée par la fixation du LPS au CD14, en coopération avec la protéine TLR4 (Toll-like receptor 4 ) et MD2. Une première voie fait intervenir deux protéines adaptatrices, MyD88 et TIRAP [11], capables de mobiliser la voie de signalisation dépendante des protéines IRAK4, TRAF6, TABl et TAKl, mais également des MAP kinases (MKK, MAP kinase kinase) et de la protéine $\mathrm{p} 38$. Cette cascade d'activations moléculaires, notamment par le biais du complexe moléculaire NEMO/ IKK $\alpha / I K K \beta$, induit la dégradation de IKB, la translocation de NFKB vers le noyau et l'activation d'APl menant à

I'activation transcriptionnelle de certains gènes codant pour des cytokines inflammatoires, telles que le TNF $\alpha$, I'IL1, I'IL8, I'IL6, I'IL12 ou encore MIPl $\alpha$. Indépendamment de TRAF6, le facteur de transcription C/EBP $\beta$ (ou NFIL6) semble être mobilisé lors de l'activation de cette voie (MyD88), par une voie de signalisation encore mal décrite, et permet notamment la production de prostaglandine $\varepsilon_{2}$ [12]. L'autre voie empruntée lors de I'activation du récepteur TLR4 fait intervenir une voie indépendante de MyD88 où interviennent deux autres protéines adaptatrices, TRAM et TRIF [13]. Ces protéines adaptatrices, au même titre que MyD88/TIRAP, activent non seulement la voie des MAP kinases et de la p38, mais également une voie qui leur est propre et qui permet la synthèse de I'IFN $\beta$, de NOS2 et de I'IP10 à la suite de l'activation du facteur de transcription IRF3 [11]. Plus spécifiquement, la voie dépendante de TLR5 est empruntée par les bactéries flagellées. Elle fait intervenir MyD88 et IRAK, induit la translocation de NFKB vers le noyau, permet l'activation de la protéine $p 38$, et aboutit à la production de cytokines telles que l'IL6 et le TNF $\alpha$. APl : adaptor protein complex ; C/EBP $\beta$ : CCAAT/enhancer-binding protein $\beta ;$ CDI4 : cluster de différenciation ; IFN : interféron ; IKK : inhibitor of $\kappa B$ kinase ; IL : interleukine ; IP10 : interferon inducible protein $10 ;$ IRAK : interleukin 1 receptor-associated kinase ; IRF3 : interferon regulatory factor 3 ; IKB : inhibitor of nuclear factor $K B$; LPS : lipopolysaccharide; MIPl $\alpha$ : macrophage inflammatory protein $1 \alpha$; MKK : MAP kinase kinase ; MyD88 : myeloid differentiation factor 88 ; NEMO : nuclear factor $\kappa B$ essential modifier; NFK B : nuclear factor $\kappa B$. TAB : transforming growth factor $\beta$-activated protein kinase-binding protein; TAK : transforming growth factor- $\beta$-associated kinase ; TIRAP : Toll-interleukin 1 receptor domain-containing adapter protein ; TLR : Toll like receptor; TNF $\alpha$ : tumor necrosis factor $\alpha$; TRAF : tumor necrosis factor receptor associated factor ; TRAM : TRIF-related adapter molecule ; TRIF : Toll/interleukin-1 receptor domain-containing adaptor protein inducing interferon $\beta$. 
chez plusieurs lignées murines : Ity pour Salmonella, Bcg pour Mycobacterium et Lsh pour Leishmania. II fallut attendre 20 ans pour rapprocher ces marqueurs de résistance à un gène unique, Nrampl [21] (natural resistance associated macrophage protein 1), nommé désormais Slcllal pour son appartenance à la famille des transporteurs ioniques. NRAMP1, exprimée par les macrophages et les polynucléaires neutrophiles, est une protéine de $56 \mathrm{kDa}$ à 12 domaines transmembranaires, fortement hydrophobe et contenant une boucle extracytoplasmique glycosylée [22].

Pendant la phagocytose, des vésicules d'endocytose particulières contenant Salmonella (SCV, Salmonella-containing vesicle) permettent aux pathogènes de survivre et de se répliquer. La fusion de tels compartiments avec les endosomes est habituellement rapide (quelques minutes), mais, grâce à la machinerie d'évitement de Salmonella, la fusion avec les endosomes tardifs s'effectue avec plusieurs heures de retard [23]. Ces endosomes contiennent de nombreuses enzymes, à l'origine notamment de la destruction des composants microbiens.

NRAMPI est recrutée dans la membrane des phagosomes et reste associée à ces membranes jusqu'à la formation des phagolysosomes, au sein desquelles elle permet l'acquisition du récepteur M6PR (mannose 6 phosphate receptor), une protéine connue pour régler le transport de certaines enzymes lysosomales (Figure 2). NRAMP1 est par ailleurs capable de transporter les ions divalents $\left(\mathrm{Fe}^{2+}, \mathrm{Zn}^{2+}, \mathrm{Mn}^{2+}\right)$ du domaine intravésiculaire vers le compartiment cytoplasmique, en fonction du $\mathrm{pH}$ vésiculaire [24]. De son côté, Salmonella possède un gène orthologue à Nrampl, mntH, dont la protéine est impliquée dans un processus similaire, mais produisant un flux d'ions divalents du domaine intravésiculaire vers le compartiment intravacuolaire bactérien. NRAMPI apparaît donc clairement comme un compétiteur naturel de l'apport aux bactéries en métabolites ioniques essentiels à leur réplication [22].

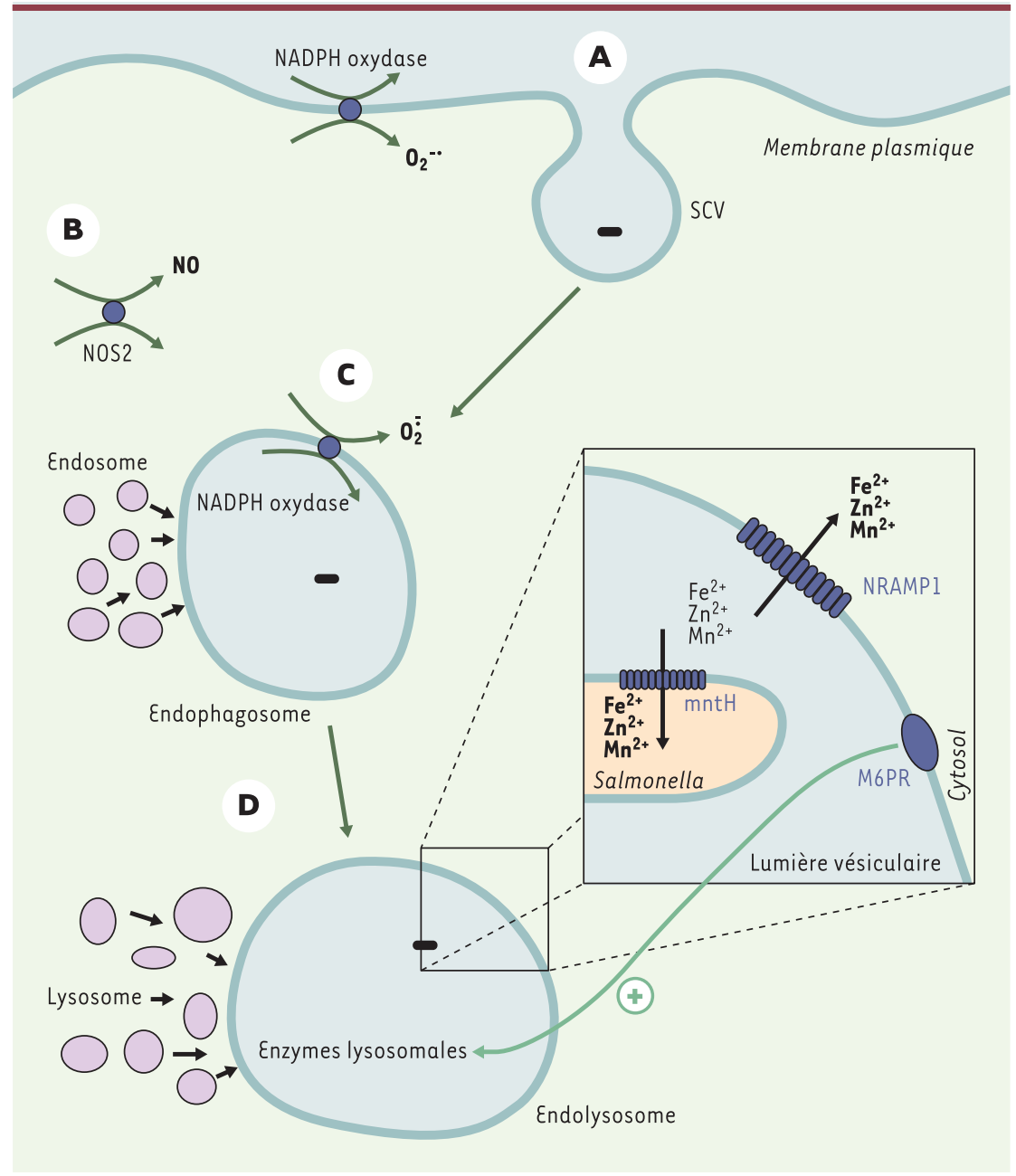

Figure 2. Acteurs moléculaires de destruction de Salmonella. À la suite de la reconnaissance du pathogène par les cellules phagocytaires mononucléées, la bactérie subit une internalisation (A) (formation des vésicules contenant Salmonella, SCV) en vue de sa phagocytose. Celle-ci est rendue possible par l'arrangement d'événements séquentiels faisant intervenir différentes molécules dont les actions combinées conduisent à la destruction de la bactérie : l'activation de la nitric oxide synthase (NOS2) (B), notamment en réponse à l'activation de TLR4 (Toll like receptor), permet la production du monoxyde d'azote (NO), diffusible au sein de la cellule, qui est délétère pour les acides nucléiques et les protéines, et inhibe la fonction mitochondriale et l'apoptose chez l'hôte. De même, l'activation de la NADPH oxydase (c) conduit à la production $\mathrm{du}$ radical

$\mathrm{O}_{2}^{-}$

qui, en présence de chlore ou de fer, présente un risque d'altération pour l'ADN, les protéines et l'intégrité membranaire bactérienne. Ces deux intervenants (NOS2 et NADPH oxydase) semblent agir à des moments différents de l'infection. Plus tard au cours du processus, les enzymes lysosomales, après fusion des phagosomes et du lysosome (D), agissent de concert pour dégrader les composants bactériens. Leur action est potentialisée par le récepteur du mannose 6 phosphate (M6PR), connu pour régler le transport d'enzymes lysosomales du réseau transgolgien aux lysosomes. L'expression de NRAMP1 (natural resistance associated macrophage protein 1) à la surface des SCV favorise l'acquisition de M6PR et la fusion entre phagosomes et lysosomes. De son côté, Salmonella exprime mntH, un transporteur des ions divalents $\mathrm{Fe}^{2+}, \mathrm{Zn}^{2+}$, $\mathrm{Mn}^{2+}$ essentiels pour sa survie; la protéine NRAMPl, exprimée dans la membrane vésiculaire de l'endolysosome, agit comme compétiteur naturel du transport de ces ions divalents. 


\section{NADPH oxydase et NOS2}

Les deux enzymes, NADPH oxydase et NOS2, participent à la défense antimicrobienne par leur action oxydante [25] (Figure 2). Leur importance en pathologie humaine a été mise en relief en étudiant des patients souffrant de maladies granulomateuses chroniques, résultant de mutations dans les différentes sous-unités de la NADPH oxydase [26]. II est acquis que la NADPH oxydase agit en transformant l'oxygène moléculaire $\mathrm{O}_{2}$ en anion superoxyde $\mathrm{O}_{2}$, lequel peut produire $\mathrm{H}_{2} \mathrm{O}_{2}$ sous l'effet de la superoxyde dismutase. En présence de chlore ou de fer, les dérivés chlorés et les radicaux hydroxyles $\mathrm{OH}^{-}$synthétisés sont capables d'agir comme des bactéricides puissants. Leur toxicité agit au niveau de l'ADN, des protéines et des lipides membranaires [27].

La synthèse du monoxyde d'azote (NO) est catalysée par la NO synthase, représentée par trois isoformes. La NOS2 est exprimée dans différents types cellulaires, notamment les macrophages, les polynucléaires neutrophiles ou encore les hépatocytes. Le NO a une fonction capitale dans le maintien de l'homéostasie vasculaire, mais joue un rôle très important dans la défense antimicrobienne, notamment en supprimant la synthèse d'ADN et la synthèse protéique du pathogène, et en inhibant la fonction mitochondriale et celle de l'apoptose chez I'hôte [28]. Ces propriétés du NO en font une molécule aux fonctions ambiguës, car ses capacités inhibitrices de la synthèse protéique peuvent, dans certains cas, être délétères pour l'organisme hôte.

L'importance de la NADPH oxydase et de la NOS2 peut être illustrée par des travaux relatant divers moyens sélectionnés par Salmonella pour échapper à la défense de l'hôte. Salmonella a développé le moyen d'inhiber la fusion vésiculaire [29], mais également de délocaliser la NADPH oxydase [30] et la NOS2 [31], avec pour effet de les détourner de leur pouvoir bactéricide.

\section{Défensines}

Les défensines font partie d'un groupe de peptides cationiques synthétisés et sécrétés notamment par les cellules de Paneth, entités spécialisées localisées au sein des cryptes de l'intestin grêle (les défensines- $\alpha$ des cellules de Paneth sont appelées cryptdines). Leur rôle dans la défense de l'hôte contre Salmonella typhimurium a été démontré à l'aide de deux modèles de souris génétiquement modifiées. Le premier modèle implique l'inactivation du gène Mmp7 (matrilysin), codant pour une enzyme nécessaire à la maturation des cryptdines chez la souris. Lors d'une infection à Salmonella typhimurium par voie orale, les souris dont le gène codant pour Mmp7 a été invalidé sont beaucoup plus sensibles à l'infection [32]. Le second modèle implique la création de souris transgéniques exprimant le gène humain HD5 (orthologue du gène codant pour la cryptdine 4 chez la souris) de façon spécifique dans les cellules de Paneth [33]. Ces souris transgéniques font preuve d'une résistance accrue à l'infection à Salmonella typhimurium administrée oralement, et non par voie parentérale. Les défensines sécrétées par les cellules de Paneth contribuent donc à la défense de l'hôte en jouant un rôle dans la survie des pathogènes invasifs tels que Salmonella.

\section{Conclusions}

La majeure partie des gènes précédemment décrits ont été mis en évidence à l'aide d'études de mutations transmises selon la règle mendélienne, en tirant parti du fait que la résistance à Salmonella peut provenir de l'absence ou de la mutation ponctuelle d'un seul gène. La connaissance des génomes humain et murin, et les techniques de biologie moléculaire actuelles, telles que l'étude des locus de traits complexes, permettent dorénavant de déceler les fonctions et les interactions de gènes distincts. II semble donc que les années à venir devraient voir émerger la connaissance de nouvelles interactions moléculaires entre Salmonella et son hôte, laissant espérer que de nouvelles approches thérapeutiques seront prochainement accessibles. $\nabla$

\section{SUMMARY}

Protagonists of innate immunity during infection with Salmonella

Salmonella are facultative intracellular Gram-negative bacteria that are found ubiquitously in nature and have the ability to infect a wide range of hosts including humans, domesticated, wild mammals, and birds. The principal clinical manifestations associated with Salmonella infection in humans are enteric fever (typhoid and paratyphoid) and a self-limiting gastroenteritis (salmonellosis). Additionally, silent carriage of this bacterium is frequent and contributes to disease dissemination. Typhoid fever still represents a major public health problem in many developing countries. On the other hand, industrialized countries experience an increased incidence of nontyphoidal Salmonella infections with most cases tracing back to food contamination. Studies using mouse model of infection with a highly virulent Salmonella typhimurium serotype have provided important insight into the complexity of the innate immune response to infection. The players are numerous but emphasis was placed on the genes that were discovered using genetic approaches and in vivo assay with live pathogen and include positional cloning of mouse mutations and manipulation of genes in the context of whole animal either by transgenesis or knockout technologies. Some of the critical genes include those known to play a role in the detection of the bacteria ( $C d l 4, L b p$, Tlr4 and Tlr5) and in microbicidal activity (Slcllal, Nos2, NADPH oxidase and cryptdins). These discoveries have already initiated the search for the contribution of particular genetic pathways in the innate immune response of humans to infection with Salmonella and other intracellular microorganisms. $\Delta$ 


\section{RÉFÉRENCES}

1. Brenner FW, Villar RG, Angulo FJ, et al. Salmonella nomenclature. J Clin Microbiol $2000 ; 38: 2465-7$.

2. Gruenheid S, Finlay BB. Microbial pathogenesis and cytoskeletal function. Nature $2003 ; 422: 775-81$.

3. Jack RS, Fan X, Bernheiden M, et al. Lipopolysaccharide-binding protein is required to combat a murine gram-negative bacterial infection. Nature 1997 ; 389 : 742-5.

4. Triantafilou M, Triantafilou K. Lipopolysaccharide recognition: CD14, TLRs and the LPS-activation cluster. Trends Immunol $2002 ; 23$ : 301-4.

5. Vogel SN, Hansen CT, Rosenstreich DL. Characterization of a congenitally LPSresistant, athymic mouse strain. J Immunol 1979; 122 : 619-22.

6. 0 'Brien AD, Rosenstreich DL, Scher I, et al. Genetic control of susceptibility to Salmonella typhimurium in mice: role of the LPS gene. J Immunol $1980 ; 124: 20-4$.

7. Hoffmann JA, Reichhart JM. Drosophila innate immunity: an evolutionary perspective. Nat Immunol $2002 ; 3$ : 121-6.

8. Poltorak A, He X, Smirnova I, et al. Defective LPS signaling in $(3 \mathrm{H} / \mathrm{HeJ}$ and C57BL/10ScCr mice: mutations in Tlr4 gene. Science $1998 ; 282: 2085-8$.

9. Qureshi ST, Larivière L, Leveque $G$, et al. Endotoxin-tolerant mice have mutations in Toll-like receptor 4 (Tlr4). J Exp Med 1999 ; $189: 615-25$.

10. Takeda K, Kaisho T, Akira S. Toll-like receptors. Annu Rev Immunol $2003 ; 21$ : 335-76.

11. Beutler B, Hoebe K, Du X, Ulevitch RJ. How we detect microbes and respond to them: the Toll-like receptors and their transducers. J Leuk Biol $2003 ; 74: 479-85$.

12. Uematsu S, Matsumoto M, Takeda K, Akira S. Lipopolysaccharide-dependent prostaglandin $\varepsilon(2)$ production is regulated by the glutathione-dependent prostaglandin $\varepsilon(2)$ synthase gene induced by the Toll-like receptor 4/MyD88/NFIL6 pathway. J Immunol 2002 ; $168: 5811-6$.

13. Yamamoto M, Sato S, Mori K, et al. Cutting edge: A novel Toll/IL-1 receptor domaincontaining adapter that preferentially activates the IFN-beta promoter in the Tolllike receptor signaling. J Immunol $2002 ; 169: 6668-72$.

14. Doyle SE, O'Connell RM, Miranda GA, et al. Toll-like receptors induce a phagocytic gene program through p38. J Exp Med $2004 ; 199: 81-90$.

15. Picard $C$, Puel $A$, Bonnet $M$, et al. Pyogenic bacterial infections in humans with IRAK-4 deficiency. Science 2003 ; 299 : 2076-9.

16. Jain $A, M a C A$, Liu $S$, et al. Specific missense mutations in NEMO result in hyper-IgM syndrome with hypohydrotic ectodermal dysplasia. Nat Immunol $2001 ; 2$ : 223-8.

17. Courtois G, Smahi A, Reichenbach J, et al. A hypermorphic IkappaBalpha mutation is associated with autosomal dominant anhidrotic ectodermal dysplasia and T cell immunodeficiency. J Clin Invest 2003 ; 112 : 1108-15.

18. Hayashi F, Smith KD, Ozinsky A, et al. The innate immune response to bacterial flagellin is mediated by Toll-like receptor 5 . Nature $2001 ; 410: 1099-103$.
19. Gewirtz AT, Navas TA, Lyons S, et al. Cutting edge: bacterial flagellin activates basolaterally expressed TLR5 to induce epithelial proinflammatory gene expression. J Immunol $2001 ; 167: 1882-5$

20. Sebastiani $G$, Leveque $G$, Larivière $L$, et al. Cloning and characterization of the murine toll-like receptor 5 (Tlr5) gene: sequence and mRNA expression studies in Salmonella-susceptible MOLF/Ei mice. Genomics $2000 ; 64: 230-40$.

21. Vidal S, Tremblay ML, Govoni G, et al. The Ity/Lsh/Bcg locus: natural resistance to infection with intracellular parasites is abrogated by disruption of the Nrampl gene. J Exp Med 1995 ; $182:$ 655-66.

22. Cellier P, Gros P. The Nramp family. New York : Landes Bioscience, 2004 : 194 p.

23. Brumell JH, Perrin AJ, Goosney DL, Finlay BB. Microbial pathogenesis: new niches for Salmonella. Curr Biol $2002 ; 12$ : R15-7.

24. Jabado N, Jankowski A, Dougaparsad $S$, et al. Natural resistance to intracellular infections: natural resistance-associated macrophage protein 1 (Nrampl) functions as a pH-dependent manganese transporter at the phagosomal membrane. J Exp Med 2000 ; 192 : 1237-48.

25. Vazquez-Torres A, Fang FC. Oxygen-dependent anti-Salmonella activity of macrophages. Trends Microbiol $2001 ; 9: 29-33$

26. Pollock JD, Williams DA, Gifford MA, et al. Mouse model of X-linked chronic granulomatous disease, an inherited defect in phagocyte superoxide production. Nat Genet $1995 ; 9: 202-9$.

27. Morel F, Doussiere J, Vignais PV. The superoxide-generating oxidase of phagocytic cells. Physiological, molecular and pathological aspects. Eur J Biochem 1991; 201 : 523-46.

28. Nussler AK, Billiar TR. Inflammation, immunoregulation, and inducible nitric oxide synthase. J Leuk Biol 1993; 54 : 171-8.

29. Uchiya K, Barbieri MA, Funato K, et al. A Salmonella virulence protein that inhibits cellular trafficking. EMBO J 1999 ; 18 : 3924-33.

30. Vazquez-Torres A, Xu Y, Jones-Carson J, et al. Salmonella pathogenicity island 2dependent evasion of the phagocyte NADPH oxidase. Science $2000 ; 287$ : 1655-8.

31. Chakravortty D, Hansen-Wester I, Hensel M. Salmonella pathogenicity island 2 mediates protection of intracellular Salmonella from reactive nitrogen intermediates. J Exp Med 2002 ; 195 : 1155-66.

32. Wilson CL, Ouellette AJ, Satchell DP, et al. Regulation of intestinal alpha-defensin activation by the metalloproteinase matrilysin in innate host defense. Science $1999 ; 286: 113-7$.

33. Salzman NH, Ghosh D, Huttner KM, et al. Protection against enteric salmonellosis in transgenic mice expressing a human intestinal defensin. Nature 2003 ; $422: 522-6$.

\section{TIRÉS À PART}

D. Malo 